First Peoples Child \& Family Review

An Interdisciplinary Journal Honouring the Voices, Perspectives, and Knowledges of

First Peoples through Research, Critical Analyses, Stories, Standpoints and Media

Reviews

\title{
Quels sont les facteurs favorisant ou inhibant la réussite éducative des élèves autochtones?
}

\section{Hélène Archambault}

Volume 5, Number 2, 2010

URI: https://id.erudit.org/iderudit/1068936ar

DOI: https://doi.org/10.7202/1068936ar

See table of contents

Publisher(s)

First Nations Child and Family Caring Society of Canada

ISSN

1708-489X (print)

2293-6610 (digital)

Explore this journal

Cite this article

Archambault, H. (2010). Quels sont les facteurs favorisant ou inhibant la réussite éducative des élèves autochtones? First Peoples Child \& Family Review, 5(2), 107-116. https://doi.org/10.7202/1068936ar

\section{Article abstract}

Depuis plus de dix ans, de nombreux changements sont survenus au sein des communautés autochtones des Premières nations du Québec en matière d'éducation. L'analyse de la situation scolaire des Autochtones doit tenir compte de certaines composantes qui leur sont propres. Comprendre ces différentes composantes structurant la réussite éducative des élèves autochtones nécessite la présentation de multiples facteurs. Dans cet article, nous présentons les facteurs favorisant et inhibant la réussite scolaire des élèves autochtones. Bien entendu, cet article ne prétend pas tracer un portrait exhaustif du parcours éducatif des élèves autochtones, ni élaborer sur toutes les dimensions existantes pour chaque nation. Il s'agit simplement de faire le point sur la réalité scolaire des Autochtones afin de mieux connaître et comprendre leur profil scolaire actuel.
This document is protected by copyright law. Use of the services of Érudit (including reproduction) is subject to its terms and conditions, which can be viewed online.

https://apropos.erudit.org/en/users/policy-on-use/ 


\title{
First Peoples Child \& Family Review
}

An Interdisciplinary Journal Honoring the Voices, Perspectives and Knowledges of First Peoples through Research, Critical Analyses, Stories, Standpoints and Media Reviews

\section{Quels sont les facteurs favorisant ou inhibant la réussite éducative des élèves autochtones?}

\author{
Hélène Archambaulta
}

${ }^{a}$ Ph.D., Professeure adjointe - Faculté d'éducation, Collège universitaire de Saint-Boniface, Winnipeg, Manitoba, Canada.

\section{Introduction}

Depuis quelques années, de nombreuses études ont démontré la présence de facteurs favorisant ou inhibant la réussite éducative des élèves en milieu autochtone. Ainsi, dans le cadre de cet article, nous présentons les différents facteurs qui ont une incidence sur le rendement scolaire des élèves des Premières nations du Québec. Les principaux facteurs favorisant la réussite scolaire des élèves en milieu autochtone sont présentés dans une première partie, suivie des principaux facteurs inhibant la réussite éducative dans une seconde partie.

\section{PARTIE I: Les principaux facteurs fa- vorisant la réussite scolaire chez les élèves autochtones}

Avec le plan d'action en matière d'éducation du ministère des Affaires indiennes et du Nord Canada (2005), les Premières nations du Québec s'engagent à offrir à leurs enfants une éducation de qualité qui leur permettra d'améliorer leur rendement scolaire. Au cours des dernières années, la mise en place des services éducatifs axés sur la réussite scolaire est devenue une priorité nationale non seulement pour les

Questions or correspondence concerning this article may be addressed to:

Hélène Archambault, Ph. D.

Professeure adjointe - Faculté d'éducation

Collège universitaire de Saint-Boniface

200, avenue de la Cathédrale,

Winnipeg, Manitoba, Canada $\mathrm{R} 2 \mathrm{H} \mathrm{OH} 7$

Téléphone: 1.204.233.0210 poste 453

Courriel: harchambault@ustboniface.mb.ca

\section{Résumé de l'article}

Depuis plus de dix ans, de nombreux changements sont survenus au sein des communautés autochtones des Premières nations du Québec en matière d'éducation. L'analyse de la situation scolaire des Autochtones doit tenir compte de certaines composantes qui leur sont propres. Comprendre ces différentes composantes structurant la réussite éducative des élèves autochtones nécessite la présentation de multiples facteurs. Dans cet article, nous présentons les facteurs favorisant et inhibant la réussite scolaire des élèves autochtones.

Bien entendu, cet article ne prétend pas tracer un portrait exhaustif du parcours éducatif des élèves autochtones, ni élaborer sur toutes les dimensions existantes pour chaque nation. II s'agit simplement de faire le point sur la réalité scolaire des Autochtones afin de mieux connaître et comprendre leur profil scolaire actuel.

Mots clés: autochtone, milieu scolaire, réussite éducative

intervenants du milieu scolaire, mais également, à une plus grande échelle, pour l'ensemble des communautés autochtones des Premières nations du Québec (ministère de l'Éducation, du Loisir et du Sport, 2005; ministère des Affaires indiennes et du Nord Canada, 2005; Assemblée des Premières Nations, 2005).

Encore de nos jours, les jeunes Autochtones qui vivent dans leurs communautés sont considérés comme étant parmi les groupes sociaux les plus vulnérables (ministère de l'Éducation, du Loisir et du Sport, 2005).

En dépit des nombreux obstacles que devront surmonter les élèves autochtones durant leur parcours scolaire, plusieurs études ont identifié divers déterminants favorisant leur réussite éducative (Assemblée des Premières Nations, 1988; Kirkness et Bowman, 1992; Wilson, 1994; Commission royale des peuples autochtones, 1996; Wotherspoon et Schissel, 1998; Hampton et Roy, 2002; Malatest et coll., 2002, ministère des Affaires indiennes et du Nord Canada, 2005). Dégagés de la littérature recensée, ces principaux déterminants sont présentés ci-après : 
priorités gouvernementales, intervention précoce, participation des parents et des aînés, relation maitre - élève, stratégies pédagogiques et établissements scolaires autochtones.

Avant d'exposer les différents déterminants, il semble essentiel de proposer une définition du concept de la réussite scolaire pour les besoins de nos travaux de recherche. Pour Gaskel (1995), ce concept a plutôt une portée élargie, il ne se résume pas à une définition ou à une formule simpliste. Létude canadienne de Wotherspoon et Schissel (1998) abonde dans le même sens :

Au-delà de l'acceptation générale et des attentes associées aux diplômes d'études, aux connaissances de base et aux normes de discipline, la définition d'un bon élève varie d'une école à l'autre, selon les attentes morales et sociales des parents, du personnel enseignant et de la collectivité élargie (Wotherspoon, Schissel, 1998, p.9).

De nombreuses recherches proposent différentes définitions du concept de la réussite scolaire, mais nous avons retenu cette dernière puisquelle correspond à notre vision en raison de sa dimension élargie et de son caractère relatif.

\section{Priorités gouvernementales}

En 2004-2005, une des priorités nationales du ministère des Affaires indiennes et du Nord Canada vise l'amélioration de la réussite scolaire de tous les enfants des Premières nations d'un bout à l'autre du pays (ministère des Affaires indiennes et du Nord Canada, 2005). Jouir des mêmes possibilités d’avenir et de la même qualité de vie que tous les enfants canadiens occupe une place proéminente à l'heure actuelle. Bien que de tout temps les élèves autochtones accusent un retard académique (ministère de l'Éducation, 2004), on enregistre d'importants progrès depuis quelques années (Malatest et coll., 2004). À cetégard, le ministère des Affaires indiennes et du Nord Canada (2005) énonce que : (...) la proportion des élèves des Premières nations vivant dans une réserve qui a terminé leurs études secondaires a toutefois augmenté de façon significative, passant de $31.4 \%$ à $41.4 \%$ en 2001 (p.3). Les divers paliers gouvernementaux sont déterminés à prendre des mesures concrètes pour que cette tendance se poursuive au cours des prochaines années (ministère des Affaires indiennes et du Nord Canada, 2005; ministère de l'Éducation, du Loisir et du Sport, 2005). Ainsi, les objectifs généraux du plan d’action en matière d'éducation d'avril 2005 du ministère des Affaires indiennes et du Nord Canada visent le rehaussement de la qualité, de l'accessibilité et de la pertinence d'enseignement et du soutien pédagogique dispensés aux élèves des Premières nations (ministère des Affaires indiennes et du Nord Canada, 2005). Pour atteindre ces objectifs, certains efforts seront nécessaires. À cet effet, les différents acteurs impliqués dans le domaine de léducation (les élèves, les parents, le personnel enseignant, les directions d'école, les communautés autochtones, les dirigeants des Premières nations, les paliers gouvernementaux) devront continuer à mettre en commun leurs forces vives, et ce, pour l'atteinte des résultats collectivement définis.

\section{Intervenir tôt}

Depuis plusieurs années, les spécialistes en santé infantile confirment que les six premières années constituent une étape cruciale du développement global de l'enfant (Dion Stout et Kipling, 1999). Hertzman (1996) a reconnu que l'implantation de programmes d'intervention précoce, auprès des jeunes enfants de milieux moins nantis, favorise leurs capacités d'apprentissage, leur socialisation et leur succès scolaire. Par l'implantation de ces programmes, l'enfant venant d'un milieu défavorisé arrive (...) à l'école avec un coffre à outils bien garni (éveil à la lecture et à l'écriture, développement du langage, habiletés sociales, etc.) (Blain, 2004, p.20).

Au cours des dernières années, les Autochtones considèrent important de bien préparer leurs enfants à l'entrée scolaire afin qu'ils obtiennent de meilleurs résultats à l'école (Commission royale sur les peuples autochtones, 1996). Dès 1988, le gouvernement fédéral appuie les initiatives communautaires destinées à la petite enfance de milieux autochtones afin de favoriser lacquisition des pré-requis avant le commencement de lécole (Commission royale sur les peuples autochtones, 1996). Ainsi, pour aider à améliorer le développement et la maturité scolaire des jeunes enfants des Premières nations du Canada, le gouvernement canadien a mis en place, en 1995, le Programme d'aide préscolaire aux Autochtones (PAPA). La culture et la langue, l'éducation, la promotion de la santé, la nutrition, le soutien social et l'engagement parental sont les principaux éléments à partir desquels des projets éducatifs sont élaborés. Cette initiative a pour but (...) d'inculquer un sens de fierté et un désir d'apprendre, d'aider à l'acquisition des compétences parentales, de favoriser le développement émotionnel et social, d'accroitre la confiance et d'améliorer les relations familiales (Santé Canada, 2002). En fait, ce programme permet d’appliquer des principes holistiques à léducation préscolaire des enfants autochtones tout en répondant à leurs besoins intellectuels, spirituels, émotionnels et physiques.

En guise d'illustration, le centre préscolaire Gengenlilas situé à plus de 320 kilomètres au nord de Victoria sur l'île de Vancouver offre aux enfants autochtones de la Première nation de Campbell River le programme d'aide préscolaire aux Autochtones depuis la fin septembre 1996. Divers aspects culturels spécifiques à cette nation y sont enseignés tels que la langue, la danse, les chants et les histoires ainsi que les légendes de Campbell River. Selon les écrits du ministère des Affaires indiennes et du Nord Canada (2004), lorsque les enfants fréquentent le milieu préscolaire avant le primaire, ils ont tendance à avoir beaucoup de succès (p.6). Une enseignante de la maternelle œuvrant auprès délèves autochtones abonde dans le même sens 
en énonçant que les élèves qui ont fréquentéle centre préscolaire Gengenlilas (...) sont plus avancés au plan scolaire que ceux qui n'ont pas fréquenté ce genre d'établissement (ministère des Affaires indiennes et du Nord Canada, 2004, p.6). Grâce à l'instauration du programme daide préscolaire aux Autochtones dans les communautés, les enfants des Premières nations possèdent une meilleure préparation à lécole favorisant ainsi un meilleur rendement scolaire (Santé Canada, 1999).

\section{Participation des parents et contribution des Aînés}

Parents et famille rapprochée sont les premiers responsables de léducation de leurs enfants, en conséquence, il est souhaitable d'accroitre leur participation pour favoriser la réussite scolaire des élèves (ministère de l'Éducation, 2001). Comme le souligne Blain: L'engagement du parent dans le parcours scolaire de son enfant est considéré comme facteur essentiel de la réussite (Blain, 2004, p.19). Dans la même perspective, le ministère des Affaires indiennes et du Nord Canada (2005) reconnait que l'engagement continu des parents représente une condition nécessaire à la réussite scolaire des jeunes autochtones.

Depuis les dernières années, les parents des Premières nations ont développé une plus grande confiance envers le système scolaire, car il est davantage administré par des autorités autochtones. Par conséquent, les parents participent plus aux activités éducatives de leurs enfants (Commission royale des peuples autochtones, 1996). Selon la revue de littérature réalisée par Kavanagh en 2002, le rendement scolaire de lélève sera positivement influencé par la confiance des parents envers l'école et leur participation.

En effet, la combinaison de ces deux composantes entraine chez lélève (...) une plus grande assiduité, des attitudes positives, de meilleures notes et une participation accrue à l'enseignement supérieur (traduction libre) (Kavanagh, 2002, p.11).

Plus récemment, les travaux de Gauthier (2005) confirment ceux réalisés par Kavanagh. Pour les besoins de l'étude, le chercheur a rencontré seize (16) étudiants de secondaire $\mathrm{V}$ fréquentant l'école Uashkaikan de la communauté innue de Betsiamites au cours de l'année 2003-2004. Plusieurs jeunes rencontrés disent être stimulés et encouragés par leurs parents. À cet effet, un étudiant énonce ce qui suit à l'égard de ses parents :

Ils sinforment de mes notes et pour savoir si tout va bien avec les professeurs. Ils nont pas manqué un bulletin. Ils viennent voir les professeurs (...)(Gauthier, 2005, p. 212).

Pour remplir sa mission, lécole a besoin de la complicité des parents et des Aînés pour transmettre les savoirs autochtones (ministère des Affaires indiennes et du Nord Canada, 2005). Lors des consultations publiques de la Commission royale des peuples autochtones de 1996, Harold Rampersad, coordonnateur des relations interraciales et communautaires de Winnipeg a proposé :

Nous devons considérer les anciens comme les gardiens des cultures autochtones et reconnaitre leur rôle dans lenseignement de la culture et de la langue dans tous les milieux scolaires et à tous les niveaux d'études (traduction libre) (Commission royale des peuples autochtones, 1996, non paginée).

Grâce à leur contribution en milieu scolaire, les Aînés deviennent des personnes-ressources pouvant appuyer, orienter, conseiller le personnel enseignant et les élèves en partageant avec eux leur sagesse, leur philosophie, leur culture, leur histoire et leurs traditions autochtones (Commission royale des peuples autochtones, 1996). À ce propos, Béatrice Medicine (1986), chercheuse Lakota, souligne que tous les intervenants du système scolaire autochtone tireront profit du lien symbolique que créeront les anciens entre-deux domaines culturels différents (traduction libre) (Medicine, 1986, p.11). Selon les écrits de Wotherspoon et Schissel (1998): Les élèves qui se sentent les plus en sécurité, les plus à l'aise et les plus enthousiastes sont ceux qui reçoivent une certaine forme d'éducation culturelle à l'école (p.16). C'est par la concertation des différents partenaires partageant la même vision en matière de réussite scolaire que les jeunes élèves autochtones pourront atteindre leurs objectifs scolaires et jouer éventuellement un rôle actif au sein de sa communauté (ministère des Affaires indiennes et du Nord Canada, 2003, 2005).

\section{Relation maître - élève}

Wang, Heartel et Walberg (1993) ont réalisé une étude longitudinale couvrant 50 ans de recherches en éducation. Cette étude publiée en 1993 intitulé What Helps Students learn? visait à identifier les facteurs les plus susceptibles d'aider l'élève à apprendre. Basée sur une analyse de comptes rendus et de chapitres de manuels, de synthèses de recherche et d'enquêtes auprès de nombreux chercheurs en éducation, cette métaanalyse de grande envergure a identifié l'enseignant comme étant le facteur ayant le plus d'influence sur l'apprentissage des élèves. Comme le souligne Gaudreau (1998): Une relation pédagogique saine conduit à une reconnaissance et à une acceptation mutuelle de chacune des personnes dans la classe (p.47). En effet, la relation maître - élève est avant tout une relation unique qui a un impact sur la qualité des apprentissages, mais également sur la santé mentale des apprenants (Gaudreau, 1998).

Brossard (1995) propose ce qui suit :

La qualité de la relation personnelle que l'enseignante noue avec l'élève facilitera l'acquisition des connaissances chez les 
élèves. Lélève apprend mieux avec une personne quill aime et qui aime (Brossard, 1995, p.21).

Reconnaissant l'ampleur des problèmes auxquels doivent faire face les élèves vivant en milieu autochtone, des chercheurs ont démontré que la relation maitre - élève permet de combler certaines carences affectives présentes chez ces derniers. À cet effet, les données empiriques issues de travaux de recherche de nombreux chercheurs (Kleinfeld, 1975; Wilson, 1994, Wotherspoon et Schissel, 1998; Goldstein, 1999; HodsonSmith, 2000, Mitchell, 2000; Hampton et Roy, 2002) ont démontré qu’une relation maître - élève basée sur la confiance, la reconnaissance culturelle et le respect mutuel aura une influence déterminante sur le succès scolaire des élèves autochtones. Par ailleurs, ces chercheurs ont tenté d'identifier la présence de certaines attitudes des enseignants favorisant la réussite scolaire chez les élèves autochtones. Ils stipulent que les enseignants qui œuvrent auprès des élèves autochtones doivent davantage développer une relation chaleureuse, adopter une attitude positive, être disponibles et entretenir un climat de classe harmonieux (Kleinfeld, 1975; Wilson, 1994; Wotherspoon et Schissel, 1998; Goldstein, 1999; HodgsonSmith, 2000, Mitchell, 2000; Berger, 2002; Hampton et Roy, 2002). De plus, l'Assemblée des Premières Nations (1988) et la Commission royale des peuples autochtones (1996) soulignent que la présence d'enseignants autochtones influence également le rendement scolaire des élèves autochtones, car ils ont généralement plus à cœur le respect des valeurs et des traditions autochtones que les enseignants non autochtones.

En somme, il importe de savoir que la reconnaissance de la diversité culturelle autochtone etla présence de relations ouvertes et bienveillantes entre enseignants et élèves sont intimement liées à la réussite scolaire en milieu autochtone (Wauters et coll,, 1989; Lipka, 1990; Campbell, 1991; Wotherspoon et Schissel, 1998, Berger, 2002).

\section{Stratégies pédagogiques convenant aux élèves autochtones}

Au cours des dernières années, l'utilisation de diverses stratégies pédagogiques auprès des élèves autochtones favorisant leur réussite éducative a été démontrée par de nombreuses recherches. Selon la Lipka (1990), Commission royale des peuples autochtones (1996), Wotherspoon et Schissel (1998), Hampton (2002) et Presseau et coll. (2005), la reconnaissance et la valorisation de la culture autochtone dans les pratiques pédagogiques des enseignants ont un impact significatif sur la réussite scolaire des jeunes autochtones. En effet, Wotherspoon et Schissel (1998) constatent
(...) que les avantages sont plus importants, tant dans les écoles provinciales et territoriales que dans les écoles des Premières nations, lorsque la culture autochtone est intégrée de manière holistique à tous les aspects du programme et de la culture scolaire (Wotherspoon et Schissel, 1998, p.11).

De toute évidence, lécole devient un lieu de croisement de cultures où lélève apprend à construire son identité culturelle individuelle (Henry-Lorcerie, 1986b). Depuis quelques années déjà, divers programmes pédagogiques axés sur la conservation culturelle autochtone ont pris forme dans les institutions scolaires destinées aux élèves autochtones d'un bout à lautre du Canada (Commission royale des peuples autochtones, 1996; Malatest et coll., 2002, 2004).

Lors des audiences publiques de la Commission royale des peuples autochtones (1996), de jeunes autochtones ont exprimé limportance de réaliser des apprentissages concrets directement en lien avec leur quotidien et leur réalité tout en respectant leur diversité culturelle. Plus récemment, des intervenants éducatifs oeuvrant au sein de communautés autochtones des Premières nations du Québec ont été rencontrés par léquipe de recherche de Presseau et coll. (2005), ces intervenants ont déclaré ce qui suit :

Ils croient quiun enseignement axé sur des apprentissages concrets, permettant de travailler à un rythme différent et requérant, un engagement actif de la part des jeunes autochtones est propice à leur réussite et à leur persévérance scolaires (Presseau, 2005, non paginée).

Par ailleurs, Sawyer (1991), Hampton et Roy (2002) reconnaissent que lapprentissage coopératif favorise lacquisition des apprentissages et la réussite scolaire chez les élèves autochtones.

We found that cooperative learning models may be the most effective methods for facilitating success of First Nation students, which is consistent with previous studies that have identified cooperative strategies as most effective for Native students (Hampton et Roy, 2002, p.12).

Pour accroître la réussite scolaire des élèves autochtones, les recommandations de la Commission royale des peuples autochtones (1996), de l'Assemblée des Premières Nations (2005) et du ministère des Affaires indiennes et du Nord Canada (2005) proposent de consacrer des ressources particulières permettant aux équipes locales d’apporter un soutien approprié aux jeunes pour qui la réussite scolaire représente un défi. $\mathrm{Au}$ cours des dernières années, des programmes de soutien destinés aux élèves autochtones ont été implantés en vue de favoriser leur réussite scolaire (Malatest et coll., 2004). L'enseignement individualisé, lappui des Aînés, la taille de la classe réduite et des ateliers éducatifs constituent des exemples de soutien dispensés 
aux étudiants autochtones (Malatest et coll. 2004). Par la mise en place de programmes de soutien, les élèves pourront développer leur estime de soi, cultiver leur goût de l'effort et améliorer leur rendement scolaire (Malatest et coll. 2004).

Pour conclure, il nous semble pertinent de présenter le programme éducatif Dene Kede Curriculum instauré dans les communautés des Premières nations des Territoires du NordOuest. Ce programme culturel reconnu officiellement par le ministère de l'Éducation de ce territoire applique diverses stratégies pédagogiques convenant aux élèves autochtones de la maternelle à la sixième année. Construit autour de quatre composantes : la terre, le monde spirituel, les autres et soi, il vise l'épanouissement del'enfant par une approche holistique. À partir de 50 modules thématiques axés sur lutilisation d'expériences culturelles, les enseignants proposent aux élèves des activités pédagogiques où l'observation, l'analyse, l'exploration, la pratique et la collaboration sont valorisées. Dans le cadre de ce programme, la réussite scolaire des élèves autochtones repose sur des apprentissages concrets et coopératifs où le soutien et l'entraide des élèves et des Aînés sont réunis autour d’objectifs communs (Commission royale des peuples autochtones, 1996).

\section{Présence d'institutions scolaires autochtones}

Point de départ des futures réformes de l'éducation des Autochtones, le rapport de 1972 intitulé Indiens Control of Indian Education (le contrôle de l'éducation des Indiens par les Indiens), recommande la prise en charge de léducation par des Autochtones. Découlant de cette recommandation, des établissements scolaires postsecondaires gérés par des gestionnaires autochtones ont vu le jour. Les travaux de Malatest et coll. (2004)(...) ont démontré que dans toutes les situations où on donne aux Autochtones le contrôle de leurs propres programmes ou établissements, ils augmentent leurs taux d'inscription et de diplomation (p.31). Au Québec, l'Institut de formation autochtone (IFAQ) est dirigé et administré par des Autochtones depuis 1982. Cet institut offre de la formation à l'ensemble des Premières nations du Québec. Par son mandat, il développe et adapte divers programmes d'enseignement et de formation répondant aux besoins spécifiques des clientèles autochtones des Premières nations du Québec.

Encourager les Premières nations à gérer de façon autonome les programmes et les services en matière déducation est une priorité du ministère des Affaires indiennes et du Nord Canada (2005). Soulignons que le plan d'action en matière d'éducation déposé en avril 2005 est composé de mesures concrètes destinées à favoriser la réussite et la persévérance scolaire de tous les jeunes autochtones. Au cours des prochaines années, l'atteinte de ces objectifs exigera un travail concerté et soutenu entre le gouvernement fédéral, les Premières nations, les provinces, les territoires et les différents partenaires clés (ministère des Affaires indiennes et du Nord Canada (2005).

\section{PARTIE II: Les principaux facteurs inhibant la réussite éducative des élèves autochtones}

Selon le ministère des Affaires indiennes et du Nord du Canada (2005), les élèves autochtones accusent un retard significatif d'instruction par rapport aux élèves non autochtones. Ces retards augmentent considérablement leur propension à manquer d'assiduité et à abandonner leurs études tôt ou tard. Pour faciliter la compréhension des facteurs inhibant la réussite éducative des élèves autochtones, un portrait statistique de lévolution de la situation scolaire de la population autochtone au Québec sera présenté dans un premier bloc, et dans un second bloc, les principaux facteurs inhibant la réussite éducative des élèves autochtones. Ces facteurs ont été identifiés et regroupés sous trois grandes catégories: les facteurs personnels, les facteurs scolaires et les facteurs psychosociaux. Notons que ces catégories sont inclusives et méritent dêtre explicitées afin de faciliter la compréhension de cette problématique multidimensionnelle.

\section{Bloc I: Portrait statistique de l'évolution de la situation scolaire de la population autochtone du Québec}

Les données statistiques du ministère de l'Éducation au Québec (2004) et du ministère des Affaires indiennes et du Nord du Canada (2001) révèlent que les élèves autochtones accusent un retard scolaire significatif dès le primaire. Selon la déclaration des effectifs scolaires du 30 septembre 2001, 84.3 p.100 des élèves québécois de l'ensemble du Québec étaient inscrits à la 6e année du primaire sans écart dâge (en plus) avec le groupe modal. Pour les élèves autochtones, 58.5 p.100 des élèves étaient inscrits en 6e année du primaire sans écart d'âge (en plus) avec le groupe modal. Les données statistiques du ministère de l'Éducation (2004) démontrent que la proportion délèves autochtones inscrits en 5e secondaire sans écart d'âge avec lâge modale nest que de 29.7 p.100 comparativement à 74.8 p.100 pour l'ensemble du Québec. Quant à l'enseignement secondaire, les études démontrent que les retards scolaires s'accompagnent bien souvent d'une augmentation de l'abandon des études. Selon le ministère de l'Éducation (2004), la diplomation en milieu autochtone est de beaucoup inférieure à la moyenne observée dans l'ensemble du Québec.

Selon les travaux de Brais (1991), le phénomène du retard à l'entrée au secondaire a un impact sur la réussite d'études au secondaire. Il note une relation entre ce phénomène et l'obtention d'un diplôme des études secondaires. Ce chercheur souligne que près des deux tiers des élèves ayant accumulé au moins un an de retard à leur entrée au secondaire abandonnent en cours de route 
et composent la moitié de tous les décrocheurs et décrocheuses. Encore de nosjours, cen'est qu'une minoritédejeunes autochtones qui ont accès à un diplôme détudes secondaires.

Une étude réalisée conjointement par Lévesque et son équipe ainsi que le Partenariat Mikimon (2001) pour le compte de Condition féminine Canada révèle que 90 p. 100 des jeunes autochtones ne terminent pas leur secondaire avant lâge de 18 ans. Selon cette étude, le parcours scolaire des jeunes des milieux autochtones se caractérise essentiellement par de nombreux déplacements et par l'interruption répétée des études. Encore aujourd'hui, le jeune autochtone se retrouve confronté à lobligation de quitter sa famille et sa communauté au moment d'entreprendre des études postsecondaires (Malatest et coll., 2002, 2004).

\section{Bloc II: Facteurs associés au parcours scolaire difficile des élèves autochtones}

\section{Facteurs individuels :}

\section{- Les différences de style d'apprentissage}

Au cours des dernières années, plusieurs hypothèses ont été formulées quant aux causes individuelles pour expliquer le faible taux de réussite scolaire des clientèles autochtones (Wotherspoon, Schissel, 1998; Larose et coll., 2001; Lavoie, 2001, Malatest et coll., 2004). Parmi ces causes inférées, soulignons les différences de style d’apprentissage entre ceux traditionnellement utilisés dans les communautés autochtones et ceux prônés par les curriculums scolaires du ministère de l'Éducation (Backes, 1993; Larose et coll., 2001; Lavoie, 2001). Ainsi, lélève autochtone présente (...) un style cognitif de type simultané plutôt que séquentiel (Lavoie, 2001, p.19). Dès son jeune âge, l'enfant autochtone acquiert des connaissances par observation et manipulation directe sur le terrain (Scaldwell, Frame et Cookson, 1984). Il apprend donc à développer un mode d'appréhension globale de la réalité (Swisher, Deyle, 1989; Backes, 1993; Lavoie, 2001). Par exemple, le jeune autochtone part avec son père et son grand-père pratiquer la trappe de petits animaux au lieu de lapprendre dans un livre.

En contrepartie, les programmes scolaires du ministère de l'Éducation semblent privilégier, de façon générale, des activités pédagogiques sollicitant davantage des habiletés intellectuelles de type séquentiel chez lélève (Backes, 1993; Lavoie, 2001).

Les travaux de Hebert, (2000), Hampton et Roy (2002), abondent dans le même sens :

Methods that are more holistic, experiential, and use the narrative mode are more consistent with traditional Aboriginal epistemology than are teaching methods that encourage sequential, objective, and analytic orientation (Hampton et Roy, 2002, p.12).
Àce titre, Lavoie (2001) déclare que (... ) le jeune autochtone est, à la base, plus ou moins adaptée à cette réalité. Conséquemment, l'écart entre son style d'apprentissage et celui privilégié par le milieu scolaire le défavorise grandement (Lavoie, 2001, p.19). En conséquence, les différences de styles d’apprentissage doivent être prises en compte dans lanalyse des facteurs contributifs au parcours scolaire difficile des élèves autochtones.

\section{Les différences linguistiques}

Depuis la fin des années 1960, on assiste à un mouvement grandissant de la revitalisation des langues autochtones (Commission royale des peuples autochtones, 1996; Secrétariat aux affaires autochtones, 1997; Lieberman, 2002). Selon le Secrétariat aux affaires autochtones (1997) Les Amérindiens et les Inuits s'expriment davantage dans leur langue en tous lieux et en toutes circonstances (p.15). Dans un tel contexte, très jeune l'enfant autochtone apprend les bases linguistiques de la langue maternelle autochtone sans toutefois la maitriser complètement avant son entrée scolaire (Lapointe, 1998). Généralement, la langue utilisée en salle de classe (le français ou l'anglais) diffère de la langue maternelle des Amérindiens (Commission royale des peuples autochtones, 1996). Selon Maria de la Luz Reyes (1992), la maitrise de la langue maternelle est nécessaire avant de faire lacquisition d'une langue seconde. Selon le ministère de l'Éducation, du Loisir et du Sport (2005) Bon nombre d'élèves autochtones doivent étudier dans une langue qui n'est pas la leur (p.5). En conséquence, les travaux de la Commission royale des Peuples autochtones (1996), de Lapointe (1998), de Lavoie (2001) et du ministère de l'Éducation, du Loisir et du Sport (2005) révèlent que les élèves autochtones sont continuellement confrontés à une compréhension limitée de la langue d'enseignement, situation qui peut engendrer des difficultés d’apprentissage et nuire à la réussite éducative de ces élèves.

\section{Facteurs scolaires}

\section{- Pénurie des enseignants}

Selon la Fédération canadienne des enseignantes et des enseignants (2000), au cours des dernières années, on assiste à une pénurie du personnel enseignant au Québec, dans l'ensemble du Canada et à l'échelle internationale. Un sondage réalisé par Vector Research (2000) pour la Fédération canadienne des enseignantes et des enseignants, démontre que trois principaux facteurs expliquent cette pénurie : 1) les départs à la retraite, 2) la présence accrue des élèves en difficulté dans les salles de classe, 3) la baisse du taux de diplomation en éducation. Par ailleurs, les données de ce sondage révèlent qưil était de plus en plus difficile d'attirer de nouveaux diplômés en éducation dans les régions rurales et éloignées. De plus, on prévoit, d'ici quelques années que cette pénurie importante 
d'enseignants soit un phénomène encore plus marqué dans les régions éloignées et isolées géographiquement. Considérant que plusieurs communautés des Premières nations du Québec sont très dispersées géographiquement, on estime que le milieu autochtone sera davantage affecté par cette pénurie d'enseignants au cours des prochaines années.

\section{- Roulement du personnel enseignant}

Les travaux réalisés par le Groupe de travail national du ministre de l'Éducation (2002) révèlent que le roulement du personnel enseignant dans les écoles des Premières nations du Québecreprésenteunélémentimportant danslacompréhension des difficultés d'apprentissage des jeunes autochtones. Le ministère de l'Éducation du Québec (2004) révèle que le personnel enseignant qui œuvre auprès des élèves autochtones des écoles des Premières nations du Québec est plus jeune et de scolarité reconnue moins élevée que le personnel enseignant des autres commissions scolaires de l'ensemble du Québec. Les plus jeunes enseignants travaillant en milieu autochtone sont davantage caractérisés par un taux de roulement élevé. La difficulté à sadapter à la culture autochtone et l'attraction exercée par les emplois en milieu urbain représentent des barrières pour retenir les jeunes enseignants à œuvrer en milieu autochtone.

\section{- Qualification des enseignants}

Dans les écoles québécoises et davantage dans les écoles des Premières nations du Québec, il est fréquent d’autoriser des personnes non qualifiées et sans diplôme à occuper des fonctions d'enseignant selon la Fédération des syndicats de l'enseignement (2005). Par exemple, en raison d'une pénurie d'enseignants qualifiés en mathématiques, des postes dans cette discipline ont dû être confiés à des personnes nayant pas les compétences requises. De plus, linsuffisance du personnel suppléant et la difficulté à trouver du personnel qualifié pour pourvoir à des postes administratifs en milieu scolaire sont également soulevées (Fédération canadienne des enseignantes et des enseignants, 2000). Pour les communautés autochtones du Québec, on présuppose que la pénurie de personnel enseignant qualifié aura une incidence négative non seulement sur la réussite scolaire, le rendement des élèves et la qualité de l'enseignement, mais également sur les perspectives d'emploi de ces derniers (ministère des Affaires indiennes et du Nord Canada, 2005).

\section{- Manque de connaissances des enseignants relatives à l'intégration de la culture autochtone}

Plusieurs enseignants qui œuvrent auprès d'élèves autochtones appartiennent à un groupe culturel et socioéconomique non autochtone. L'Assemblée des Premières Nations (1988) stipule: Many teachers are not actively participating in the First Nation community they are serving (p.114). Selon de nombreuses études, la plupart des enseignants non autochtones connaissent peu la culture, les traditions et les valeurs fondamentales autochtones; ils ne reconnaissent pas la diversité culturelle et la complexité des besoins éducatifs des élèves de communautés autochtones (Assemblée des Premières Nations, 1988 ; Lipka, 1990; Commission royale des peuples autochtones, 1996; Wotherspoon et Schissel, 1998; Berger, 2002, Malatest et coll., 2002, 2004). Le ministère de l'Éducation, du Loisir et du Sport (2005) abonde dans le même sens :

Au cours des dernières années, bon nombre d'enseignantes et enseignants se sont dits dépourvus quant au type d'intervention à mettre en place pour répondre aux besoins de ces élèves. Leur spécificité culturelle et linguistique rendent souvent inefficaces les interventions pédagogiques courantes (ministère de l'Éducation, du Loisir et du Sport, 2005, p. 5).

Comme le souligne l'Assemblée des Premières Nations (1988): Most teachers reported that they need training in cultural awareness and community orientation (p.114). Par ailleurs, Wotherspoon et Schissel, (1998) énoncent :

Les écoles qui ne reconnaissent ni n'acceptent la culture autochtone dans leur programme, programmes d'etudes ou personnel enregistrent des taux de décrochage plus élevés, un nombre d'échecs scolaires accrus et un mécontentement à l'égard de l'instruction de la part des élèves autochtones, et risquent de rompre les liens avec les collectivités autochtones (Wotherspoon et Schissel, 1998, p.10).

En conséquence, pour favoriser la réussite éducative des élèves autochtones, les enseignants devront connaitre les valeurs, les traditions et les questions d'identité autochtones afin de les intégrer dans leurs pratiques pédagogiques (Commission royale des peuples autochtones, 1996; Malatest et coll., 2004).

\section{Facteurs psychosociaux}

Un bon nombre de jeunes des communautés des Premières nations du Québec vivent dans des conditions difficiles. Plusieurs sont aux prises avec des problèmes sociaux endémiques comme la pauvreté, le manque de soutien parental, la violence familiale, les problèmes de santé, la toxicomanie et l'alcoolisme, les logements misérables et les familles dysfonctionnelles (Ross, 1992; Santé et Bien-être social Canada, 1992; Wotherspoon et Schissel, 1998; Greenall et Loizides, 2001; Larose et coll., 2001; Lavoie, 2001). Le manque de perspective en matière d'emploi, le haut taux de chômage au sein des communautés autochtones n'ont rien pour accroître leur motivation à poursuivre leurs études (Gauthier, 2005). De plus, les valeurs sociales marquées par la compétition, l'individualisme et le pouvoir de l'argent contribuent également à dévaloriser l'éducation scolaire. En 
résumé, ces divers problèmes sociaux vécus difficilement par les jeunes, représentent des obstacles majeurs pour améliorer leur réussite éducative.

\section{Conclusion}

Dans une première partie de cet article, nous avons présenté les principaux facteurs susceptibles de favoriser le rendement et la persévérance scolaires des élèves autochtones. Les priorités gouvernementales, lintervention précoce, la participation des parents et des aînés, la relation maître - élève, les stratégies pédagogiques et les établissements scolaires autochtones sont tous des facteurs associés de près à la réussite éducative des jeunes autochtones. Depuis plusieurs années, de nombreux chercheurs se sont intéressés à identifier les différents facteurs associés au parcours difficile des élèves autochtones.

Dans la seconde partie de cet article, nous avons abordé les différents facteurs inhibant la réussite scolaire des élèves vivant en milieu autochtone. Un portrait statistique de lévolution de la situation scolaire de la population autochtone du Québec est présenté dans un premier bloc. Ensuite, dans un second bloc, différents facteurs ont été identifiés, répartis dans trois grandes catégories (les facteurs individuels, les facteurs scolaires et les facteurs psychosociaux) puis expliqués.

Au cours des prochaines années, les dirigeants autochtones en concertation avec les différents ministères provinciaux et territoriaux devront élaborer et mettre en œuvre des stratégies d'intervention visant à créer et à maintenir des conditions favorables à la réussite éducative de tous les jeunes Amérindiens afin de leur assurer un avenir plus prometteur.

\section{Références bibliographiques}

Assemblée des Premières Nations. (1988). Tradition and Education: Towards a Vision of Our Future. Summerstown, ON and Ottawa, ON: National Brotherhood/Assembly of First Nations.

Assemblée des Premières Nations. (2005). Plan d'action des Premières nations sur l'éducation. Document électronique téléaccessible à l'URL: http:// www.afn.ca/cmslib/general/education-f.pdf

Backes, J.S. (1993). The american indian high school dropout rate : a matter of style? Journal of American Indian Education, 32 (3), pp. 16-29.

Berger, P. (2002). Adaptations of Euro-Canadian Schools to Inuit Culture in Selected Communities in Nunavut. Papier présenté à la conference annuelle Arctic Research Consortium of the United States (ARCUS) et Arctic Forum, 14 th, Arlington, VA, Mai.

Blain, F. (2004). Le programme Famille, École, Communauté : Réussir ensemble. Vie pédagogique, 133, 19-20.

Brais, Y. (1991). Retard scolaire au primaire et risque d'abandon scolaire. Direction générale de la recherche. Québec: Ministère de léducation.

Brossard, L. (1995). Avoir le goût du défi- Table ronde avec des enseignants et des enseignantes du primaire. Vie pédagogique, 95, 21-23.
Campbell, M. L. (1991). Teachers' Perceptions of Learning Styles and Teaching Stratégies in Relation to Children of Indian Ancestry. Unpublished M.Ed. Thesis, University of Regina.

Canadian Education Statistics Council. (1996). Education Indicators in Canada. Toronto : Canadian Education Statistics Council.

Commission royale sur les peuples autochtones. (1996). Rassembler nos forces: le plan d'action du Canada pour les questions autochtones. Gouvernement du Canada.

De La Luz Reyes, M. (1992). Challenging Venerable Assumptions: Literacy Instruction for Linguistically Different Students. Harvard Educational Review, 1992, vol.62, no 4, p.427.

Dion Stout, M., Kipling, D., G. (1999). Nouvelles priorités concernant la santé des enfants et des jeunes Inuits et des Premières nations. Document de travail préparé pour Politiques des programmes, Secrétariat du transfert et planification- Direction générale des services médicaux, Santé Canada. Ottawa.

Fédération des syndicats de l'enseignement. (2005). Communication personnelle avec un conseiller de lorganisme (A. Larose) en date du 14 juillet 2005 .

Fédération canadienne des enseignantes et enseignants. (2000). Profil démographique de la profession enseignante. Actes du colloque juillet 2000.

Gaudreau, J. (1998). Croissance de l'enfant et école primaire. Montréal: Gaëtan Morin.

Gauthier, R. (2005). Le rapport à l'institution scolaire chez les jeunes amérindiens en fin de formation secondaire: Contribution à la compréhension du cheminement scolaire chez les Autochtones. Université du Québec à Chicoutimi.

Gaskel,J. (1995). Secondary Schools in Canada: The National Report of Exemplary Schools Project. Toronto: Canadian Education Association.

Goldstein, S. (1999). The relational zone: The role of caring relationships in the co-construction of mind. American Educational Research Journal, 36(3), 647-673.

Gouvernement du Québec (2005). Réussite éducative des élèves autochtones. Québec: Ministère de l'éducation, du loisir et du sport.

Gouvernement du Québec (2004). L'éducation des populations scolaires dans les communautés autochtones du Québec. Bulletin statistique de léducation. Québec: Ministère de léducation.

Gouvernement du Québec (2003). Attirer, former et retenir des enseignants de qualité au Québec. Québec: Ministère de l'Éducation.

Gouvernement du Québec (2001a). La formation à l'enseignement. Les orientations, les compétences professionnelles. Québec: Ministère de l'Éducation, Direction de la formation initiale du personnel enseignant.

Gouvernement du Québec (2001b). Programme de formation de l'école québécoise. Éducation préscolaire et enseignement primaire. Québec: Ministère de l'Éducation.

Gouvernement du Québec (1997b). L'école tout un programme. Énoncé de politique éducative. Québec: Ministère de l'Éducation.

Gouvernement du Québec (1997c). Prendre le virage du succès. Plan d'action ministériel pour la réforme de l'éducation. Québec: Ministère de l'Éducation.

Greenall, D., Loizides, S. (2001). Un monde numérique: Espoirs pour les Autochtones - Répondre aux besoins des Autochtones en matière d'acquisition du savoir grâce aux technologies d'apprentissage. Le Conference Board du Canada. 


\section{First Peoples Child \& Family Review, Volume 5, Number 2, 2010}

Groupe de travail national du ministre sur léducation (2002). Nos enfants. Gardiens du savoir sacré. Rapport final. Hull-Ottawa: Ministère des Affaires indiennes et du Nord Canada.

Hampton, M. (1993). Toward a redefinition of American Indian/Alaska Native education. Canadian Journal of Native Education, 20 (2), 261-309.

Hampton, M., Roy, J. (2002). Strategies for facilitating success of First Nations students. The Canadian Journal of Higner Education, 32 (3), pg. n/a.

Hardy, M. (1999). Pratiquer à lécole une pédagogie interactive. Revue Française de Pédagogie, 129, Oct.Nov.Déc., 17-28.

Hebert, Y. (2000). The state of arboriginal literacy and language education in Castellano, M.B., Davis, L. \& Lahache, L., Arboriginal education: Fulfilling the promise (pp.55-75). Vancouver, BC: UBC Press.

Henry-Lorcerie, F. (1986b). Les dessous d'une crise: crise identitaire, crise institutionnelle, crise théorique dans J.-R Henry (éd.), Nouveaux enjeux culturels au Maghreb, Annuaire de l'Afrique du Nord, CNRS, Paris.

Hertzman, C. (1996). Child Developement and Long-term Outcomes: A population Health Perspective and Summary of Sucesseful Interventions. Document de travail no. 4. Toronto: Institut canadien des recherches avancées.

Hodgson-Smith, K.L. (2000). Issues of pedagogy in Arboriginal education in Castellano, M.B., Davis, L. \& Lahache, L., Arboriginal education: Fulfilling the promise (pp.55-75). Vancouver, BC: UBC Press.

Kavanagh, B. (2002). The role of parental and community involvement in the success of First Nations learners: A review of the literature.

Kirkness, V.J., Bowman, S. (1992). Les écoles des Premières nations: Luttes et triomphes. Toronto : Association canadienne de l'éducation.

Kleinfeld, J. (1975). Effective teachers of Eskimo and Indian students. School Review, 83, 301-345.

Lapointe, L. (1998). Rapport d'évaluation des élèves de l'école Olamen. Conseil des Montagnais de la Romaine. Septembre.

Larose, F., Bourque, J., Terrisse, B., Kurtness, J. (2001). La résilience scolaire comme indice dacculturation chez les autochtones: bilan de recherches en milieux innus. Revue des sciences de l'éducation, 27, 1, pp. 151-180.

Lavoie, C. (2001). La réussite scolaire des jeunes autochtones. Document électronique téléaccessible à l'URL : http//www.ordrepsy.qc.ca/ pdf/Publications/Psyqc_Archives/juillet\%202001_Autochtone/ ArtDossier_Reussite_juillet01.pdf.

Lévesque, C., Trudeau, N., Bacon,J., Montpetit, C., Cheezo, M.-A., Lamontagne, M., Sioui Wawanoloath, C. et Le Partenariat Mikinon. (2001). Les femmes autochtones et l'emploi: défis et enjeux des programmes d'employabilité au Québec. Document électronique téléaccessible à l'URL : http// www.cfcswc.gc.ca/pubs/0662654889/index_f.htlml.

Lieberman. A.E. (2002). Se sentir partie prenante: Renforcer les cultures autochtones et les langues vernaculaires par l'entremise des TIC. Document électronique téléaccessible àl'URL: http://www.learlink.aed.org/Publications/ francais/concept_papers/Se_sentir_partieprenante.pdf.

Lipka, J. (1990). Integrating cultural formand content in one Yup'ik Eskimo classeroom : a case study. Canadian Journal of Native Education, 17, (2).

Malatest \& Associates Ltd. (2004). La population autochtone et l'éducation postsecondaire - Ce que les enseignants on appris. Montréal : Fondation canadienne des bourses détudes du millénaire.

Malatest \& Associates Ltd. (2002). Pratiques exemplaires permettant d'accroitreles taux de scolarisation postsecondaire des autochtones. Rapport préparé pour le Conseil des ministres de l'Éducation (Canada).Victoria.
Medicine, B. (1986). My Elders Tell Me. Indian Education in Canada, volume 2, The Challenge, sous la direction de Jean Barman, Yvonne Hébert et Don McCaskill, Vancouver: University of British Columbia Press.

Ministère des Affaires indiennes et du Nord Canada. (2005). Vers un ressourcement- Léducation: La formation des maitres dans les collectivités. Document d'information. Ottawa: Gouvernement du Canada.

Ministère des Affaires indiennes et du Nord Canada. (2005). Plan d'action national pour les enfants. Document d'information. Ottawa: Gouvernement du Canada.

Ministère des Affaires indiennes et du Nord Canada. (2005). Plan d'action en matière d'éducation. Ottawa: Gouvernement du Canada.

Ministère des Affaires indiennes et du Nord Canada. (2004). Le centre préscolaire Gengenlilas: un effort de la collectivité pour le bénéfice de la collectivité. Ottawa : Gouvernement du Canada.

Ministère des Affaires indiennes et du Nord Canada. (2003). Programme d'éducation - Rapport. Ottawa: Gouvernement du Canada.

Ministère des Affaires indiennes et du Nord Canada. (2000). Rassembler nos forces- Investir dans la réforme de l'éducation 1999-2000 - Fiches documentaires. Ottawa: Gouvernement du Canada.

Ministère des Affaires indiennes et du Nord Canada. (2002). Rassembler nos forces- Investir dans la réforme de l'éducation, des exemples de collectivités. Ottawa: Gouvernement du Canada.

Mitchell, H. (2000). Teaching from Arboriginal perspective. Regina, SK: University of Regina, Teaching Development Centre.

Presseau, A., Martineau, S., Bergevin, C., Prud'homme, L, (2005). Réflexion sur la formation universitaire des Autochtones. Document électronique téléaccessible à l'URL: http://www.pedagogie2005.ec-lille.fr/ resume $/ 72$.pdf.

Presseau, A., Martineau, S., Bergevin, C., Dragon, J.-F. (2005). La réussite et la persévérance scolaires de jeunes autochtones : quelles interventions pédagogiques ? Présentation au colloque de l'AQETA, 7 avril 2005.

Ross, D. P. (1992). L'éducation: un investissement pour les indiens vivant dans les réserves. Les causes du faible niveau de scolarité et les avantages économiques liés à une amélioration de léducation. Ottawa : Conseil canadien de développement social.

Santé Canada. (2002). Programme d’aide préscolaire aux Autochtones. Document électronique téléaccessible à l'URL: http://www.hc-sc-gc $\mathrm{ca} /$ fnihb-dgspni/pc/papa/introduction.htm.

Santé Canada. (1999). Programme d'aide préscolaire aux Autochtones- Cadre d'évaluation nationale. Ottawa.

Santé et Bien-être social. (1992). La santé des autochtones au Canada. Direction générale des services médicaux. Ottawa.

Sawyer, D. (1991). Native learning styles: Shorthand for instructional adaptations? Canadian Journal of Native Education, 18(1), 99-105.

Scaldwell, W.A., Frame, J. E., Cookson, D.G. (1984). Individual Intellectual Assessment of Chippewa, Muncey and Oneida Children Using The WISC-R. Papier présenté à la conference MOKAKIT, Université de Western Ontario. Ontario : London.

Secrétariat aux affaires autochtones. (1997). Les Amérindiens et les Inuits du Québec Onze nations contemporaines. Gouvernement du Québec.

Swisher, K., Deyle, D. (1989). The styles of learning are different, but the teaching is just the same: Suggestions for Teachers of American Indian youth. Journal of American Indian Education, Special Issue, August, 1-11. 


\section{Quels sont les facteurs favorisant ou inhibant la réussite éducative des élèves autochtones?}

Wang, M.C., Haertel, G. D, Walberg, H.J. (1993). Toward a knowledge base for school learning. Review of educational research, 63(3), 249-295.

Wauters, J., Bruce, J., Black, D. (1989). Learning Styles: A Study of Alaska Native and Non-Native Students. Journal of American Indian Education, Special Issue, 53-62.

Wilson, P. (1994). The professor/student relationship: Key factors in minority student performance and achievement. Canadian Journal of Native Education, 14(2),305-307.

Wotherspoon, T., Schissel, B. (1998). Marginalisation, décolonisation et voix: les perspectives de l'éducation des Autochtones au Canada. Ottawa: Document de discussion. Programme canadien de recherche en éducation. Conseil des ministres de l'éducation du Canada. 